\title{
ZUM TRANSKRIPTIONSVERFAHREN HIAT-DOS - EINE BÜNDIGE BESCHREIBUNG
}

\author{
Sevgi Dereli
}

\section{Zusammenfassung}

In dem folgenden Artikel geht es um die Darstellung des HIATTranskriptionssystems, das von Ehlich/Rehbein entwickelt wurde und sich als Verfahren zur Untersuchung von diskursanalytischen Zwecken etabliert hat. Vorangestellt der HIAT-Beschreibung sind die Entwicklungen in der gesprochenen Sprache in Etappen und die knappe Einführung der Ansätze Konversationsanalyse, Sprechakttheorie und linguistische Pragmatik. Gemeinsam ist den Ansätzen der Konversationsanalyse und der linguistischen Pragmatik, dass beide mit authentisch-empirischen Sprachdaten arbeiten. Unterschiede ergeben sich bei der Umsetzung des Gesprochenen in Schriftzeichen, was hier zu beschreiben ist.

Schlüsselwörter: Gesprochene Sprache, Mündlichkeit vs. Schriftlichkeit, Konversationsanalyse, Sprechakttheorie, linguistische Pragmatik, Transkriptionssysteme, Phonetische Transkripte, Diskurstranskripte, HIATDOS.

\section{HIAT-DOS ÇEVRIYYAZI SISTEMI}

$$
\ddot{O}_{z e t}
$$

Bu makalede ele alınan konu konuşma dilinin çözümlemesinde kullanılmak üzere Ehlich ve Rehbein tarafindan geliştirilmis çevriyazı sistemi olan HIAT'ın tanıtımını ve diğer çevriyazı sistemlerinden (örneğin GAT) farkını ortaya koymak için ele alınmış bir tanıtım yazısıdır. Bu çerçevede kisaca konuşma dilinin gelişim evreleri ve bu alanda oluşmus konuşma analizi (Konversationsanalyse), sözeylem-teorisi (Sprechakttheorie) ve kullanımbilimsel çözümleme (linguistische Pragmatik) gibi teorilere de kısaca değinilmiştir.

Anahtar sözcükler: Konuşma dilinin evreleri, konuşma analizi, sözeylem teorisi, dilbilimsel pragmatizm, transkripsiyon sistem, HIAT-DOS. 


\section{Einleitung}

Mit der Etablierung der gesprochenen Sprache in den 60er Jahren im deutschen Sprachraum wurde sie Untersuchungsgegenstand linguistischer Ansätze, in deren Zentrum das Gesprochene stand. In dieser Zeit kam es zur ersten empirischen Untersuchungen der gesprochenen Sprache. Den Schwerpunkt der gesprochenen Sprache bildeten lexikalische und syntaktische Phänomene. Dies resultierte daraus, dass gesprochene Sprache immer im Hinblick auf die geschriebene Sprache analysiert wurde; Mündlichkeit wurde mit Schriftlichkeit kontrastiert. ${ }^{1}$ In den 70er Jahren ging es dann um die Erfassung der Strukturiertheit der gesprochenen

Sprache sowie die Analysen von Dialogabläufen. In der Folgezeit, Anfang der 80er Jahre, gewann die Sprache unter dem Aspekt ihrer Handlungsqualität einen anderen Stellenwert. Es entstanden unterschiedliche Konzepte, die sich mit Gesprochenem auseinandersetzten.

Mit Analyse von Alltags- und Telefongesprächen beschäftigte sich die Konversationsanalyse (KA), die aus der Ethnomethodologie und der amerikanischen Conversation Analysis hervorging und im deutschen Raum Anfang der 70er Jahre bekannt wurde. Sie befasst sich primär mit Organisation der verbalen Interaktion, stellt die Prinzipien für die Gesprächseröffnung und -beendigung, den turn-Wechsel sowie Reparaturmechanismen, die als Zeichen von misslungener Kommunikation oder 'Kommunikationsstörungen' betrachtet werden, in den Mittelpunkt. Das Gespräch ist quasi das Ergebnis eines Organisationsprozesses, der erst im Gespräch von Gesprächspartnern schrittweise etabliert wird. Die Konversationsanalyse beschränkt, vereinfacht gesehen, ihre Untersuchung auf die Frage, wie Gespräche organisiert sind, wie sie ablaufen und wie der Sprecherwechsel zustande kommt und stellt Regeln für Ablauforganisationen bereit. ${ }^{2}$ Hingegen betont der sprachphilosophisch orientierte Ansatz aus dem angelsächsischen Raum, die Austinsche und Searlsche Sprechakttheorie, den Handlungscharakter der Sprache. Sie zeigen anhand von Sätzen auf welche Weise zahlreiche Handlungen wie Versprechen, Auffordern, Ratgeben, Warnen oder Drohen realisiert werden. Rezipiert wurde die Sprechakttheorie in Deutschland von Dieter Wunderlich und Utz Maas. In der kritischen Auseinandersetzung mit ihrer Satz- und Sprecher-Zentriertheit etablierte sich

\footnotetext{
Man kann sagen, dass die gesprochene Sprache schriftlich konzipiert wurde. Alles, was in die Schriftlichkeit und vor allem in Grammatiken aufgenommen wurde, führte zu Abstraktionsprozessen in der gesprochenen Sprache. D.h. was nicht Gegenstand der Grammatiken war, wurde auch nicht als relevantes Untersuchungsobjekt in der gesprochenen Sprache behandelt. Bestandteile der mündlichen Kommunikation wie Interjektionen, Partikeln oder tonale Strukturen waren z.B. lange kein Thema in mündlich-linguistisch angelegten Untersuchungen.

2 S. in Detail Iványi 1988, Becker-Mrotzek 1994.
} 
dadurch die linguistische Pragmatik (Vertreter: Wunderlich, Ehlich, Rehbein, Redder). Somit steht die linguistische Pragmatik mit der Diskursanalyse ${ }^{3}$ im engen Zusammenhang. In der linguistischen Pragmatik werden Diskurse nicht in Form einzelner Sätze, losgelöst von ihrem Handlungszusammenhang untersucht; vielmehr steht ihre Zweckcharakteristik als zentrale Kategorie im Mittelpunkt:

Sie versteht Diskurse als grundlegend soziale Handlungen, die in und durch gesellschaftliche Handlungserfordernisse zustande kommen [...]. Bei ihren einzelnen Handlungen machen Sprecher Gebrauch von gesellschaftlich ausgearbeiteten kommunikativen Formen, die durch Zwecke bestimmt sind, denen sie dienen.

(Becker-Mrotzek 1994:89)

Allen gemeinsam ist diesen Ansätzen (Gesprächsanalyse, Konversationsoder Diskursanalyse), dass sie aufgrund der auditiven und visuellen Aufzeichnungen mit authentischen Daten arbeiten und nach der Erhebungsphase die mündlichen Sprachdaten, je nach dem Ansatz und hinsichtlich ihrer Fragestellungen, in schriftlicher Form zur Analyse bereitstellen.

\section{Transkriptionssysteme}

Je nach dem, welche Ziele man in einer Untersuchung verfolgt, kommen unterschiedliche Transkriptionsverfahren zum Einsatz.

Die phonetische Transkription mit dem 'Internationalen Phonetischen Alphabet' (IPA) hat sich in Phonologie und Phonetik etabliert und wird bei phonetischen oder phonologischen Fragestellungen verwendet. ${ }^{4}$ Mit phonetischen Transkriptionen werden mündliche Besonderheiten in einer face-to-face Kommunikation wie Unterbrechungen oder Überlappungen nicht erfasst. Ferner sind phonetische Transkripte viel aufwendiger als Diskurstranskripte, weshalb sie sich für das Transkribieren von Diskursen nicht eignen.

'Diskurstranskriptionen' ${ }^{5}$ erstellt man mit dem gleichem Ziel wie IPA; der Flüchtigkeit der gesprochenen Sprache entgegen zu wirken. Verwendet werden sie beinahe in allen Disziplinen wie in der Soziolinguistik, der Spracherwerbsforschung oder in den Nachbardisziplinen Pädagogik oder Psychologie. Im Gegensatz zum phonetischen Transkriptionssystem sind sie durch eine einfache Lesbarkeit gekennzeichnet. Jedes Transkriptionssystem benutzt unterschiedliche Notationen. Die konversationsanalytische Transkription, im Vordergrund die systematische Beschreibung des Sprecherwechsels, verwendet die literarische Umschrift, verzichtet auf

3 Entstanden sind hier viele auf Empirie basierende Arbeiten in Institutionen. Um einige zu nennen: Ehlich/Rehbein 1986, Redder 1984 zur schulischen Kommunikation, Hoffmann 1989 zur Kommunikation im Gericht, zur Arzt-Patienten-Kommunikation s. Löning/Sager 1986, Rehbein 1986, zur interkulturellen schulischen Kommunikation s. Grießhaber 1987.

4 S. detailliert Ehlich/Switalla 1976

Zu Verschriftungen in verschiedenen Ansätzen s. Ehlich/Switalla 1976, Schlobinski 1996, §3 
Partiturschreibweise und jeder neue Sprecherbeitrag macht Gebrauch von einer neuen Zeile. Die charakteristischen Elemente für die mündliche Kommunikation wie die intonatorische Markierung von 'Hörersignalen' oder nonverbalen Elementen bleiben bei konversationsanalytischen Transkripten unberücksichtigt.

Das Verfahren GAT-Gesprächsanalytisches Transkriptionsverfahren wurde 1998 entwickelt. Es stellt den Versuch dar, 'sich auf eine verbindliche Notationskonvention zu einigen, um ein schnelles Lesen und Verstehen auch von 'Fremdtranskripten' zu gewährleisten und den Austausch von Materialien zu fördern.' (Knöbl/Steiger 2004) Auch GAT benutzt die literarische Umschrift, verzichtet auf Sonderzeichen und richtet sich nach einer sequentiellen und vertikalen Schreibweise. Das dritte Verfahren, das HIAT-Transkriptsystem unterscheidet sich von den hier angeführten Systemen u.a. durch seine Partiturschreibweise. Was das bedeutet, wird im Folgenden detailliert erörtert.

\section{Das Transkriptionsverfahren HIAT-DOS}

HIAT als ein weiteres Verfahren zur Umsetzung der gesprochenen Sprache in die Schrift wurde 1976 von Ehlich (Universität München) und Rehbein (Universität Hamburg) entwickelt. Erstellt wird HIAT per Computer im Betriebssystem MS-DOS. ${ }^{6}$ Die Abkürzung HIAT steht für halbinterpretative Arbeitstranskription. Weil der Transkribent beim Transkribieren hinsichtlich des Gehörten eine Entschei-dung fällt, ist sie halbinterpretativ. Aufgrund ihrer Modifizierbarkeit bezüglich spez-ifischer Untersuchungsfragen ist HIAT zugleich eine Arbeitstranskription. HIAT gibt die produzierte und nicht die standardisierte Form einer sprachlichen Einheit in literarischer Umschrift wieder, weshalb es zur orthographischen Abweichung in der jeweiligen Sprache kommen kann. D.h. kommt es zu einer Äußerung wie 'wir ham', so wird das nicht orthographisch in 'wir haben' wiedergegeben, sondern man hält sich an die Aussprache. Auch im türkischsprachigen Korpus 'Migrantensozialberatung-inter- kulturelle Kommunikation in Institutionen' (SoMiK) wurde dieses Prinzip beibehalten: bi anstatt bir, yau anstatt yahu oder bi daa anstatt bir daha sind als Beispiele anzuführen. ${ }^{7}$ Folgende Sonderzeichen (s.u. die Tabelle) im HIAT werden verwendet, um z.B. Paraverbalia wie Betonung, Lautstärke oder Tonhöhenverlauf zu markieren:

Zur technischen Voraussetzungen s. http://www.daf.uni-muenchen.de /HIAT/HIAT.HTM. Vgl. auch Dereli/Kanz/Scheiter 1998, Schneider 2001.

Eine orthographische Umsetzung würde zur Eliminierung der Besonderheiten wie Dialektfärbungen etc. in der gesprochenen Sprache führen. 


\begin{tabular}{|c|c|c|}
\hline Zeile des Transkripts & Sonderzeichen & Erläuterung \\
\hline \multirow{10}{*}{ Verbalzeile } & $(\quad)$ & akustisch unverständlich \\
\hline & (nee) & Hörvermutung \\
\hline & $((\quad))$ & Phänomenbeschreibung \\
\hline & 1 & Abbruch \\
\hline & $\cdot$ & Pause von 0,5 Sekunden \\
\hline & .. & Pause von 1,0 Sekunden \\
\hline & $\cdots$ & Pause von 1,5 Sekunden \\
\hline & $((2 s))$ & Pause von 2 Sekunden \\
\hline & $\underline{\text { nein }}$ & betont \\
\hline & $\begin{array}{l}\text { wiedder } \\
\text { (daa) }\end{array}$ & gedehnt \\
\hline \multirow{2}{*}{ Nonverbalzeile } & $<>$ & Phänomenbeschreibung \\
\hline & $<*---->$ & Phänomenbeschreibung am linken Rand \\
\hline \multirow{5}{*}{ Intonationszeile } & / & steigende Intonation \\
\hline & 1 & fallende Intonation \\
\hline & V & fallend-steigender Intonationsverlauf \\
\hline & $\wedge$ & steigend - fallende \\
\hline & - & gleich bleibende Intonation \\
\hline
\end{tabular}

Tab. 1: Im HIAT verwendete Notationszeichen

Ein weiteres wichtiges Merkmal der HIAT-Transkription ist ihre Partiturschreib-weise, in der die Wiedergabe der simultanen und sukzessiven Abfolge der Äußerungen von zwei und mehreren Interaktanten erfasst wird. Die durch Partiturklammer zusammengesetzten Zeilen heißen Partiturfläche. Diese sind bei Analysen für Verweise von Relevanz (s. dazu TR-Beispiel 1, Fl. 1):

Name und Verfahren orientieren sich an einer Problemlösung für die Präsentation von Simultaneität, wie sie in der Musik ausgebildet wurde. Wie dort die verschiedenen Instrumente jeweils eine eigene Zeile haben und die Töne, die sie spielen, in diesen Zeilen notiert sind, so daß auf der Vertikalen abzulesen ist, welche Töne der Einzelinstrumente gleichzeitig $\mathrm{zu}$ hören sind, genau so erhält bei der Partiturschreibweise jede(r) SprecherIn eine eigene (Partitur-)Zeile, auf der transkribiert ist, was er/sie sagt während der Zeit, die eine Zeile räumlich von links nach rechts wiedergibt. Gleichzeitiges Reden mehrerer Sprecher wird dadurch ebenso exakt darstellbar wie überlappender Beginn oder wie Hörertätigkeiten, während der Sprecher den turn behält. (http://www.daf.uni-muenchen.de/HIAT/HIAT.HTM.) 
Transkriptbeispiel 1: Erläuterungen des HIAT-Transkripts (TR-12BT8)
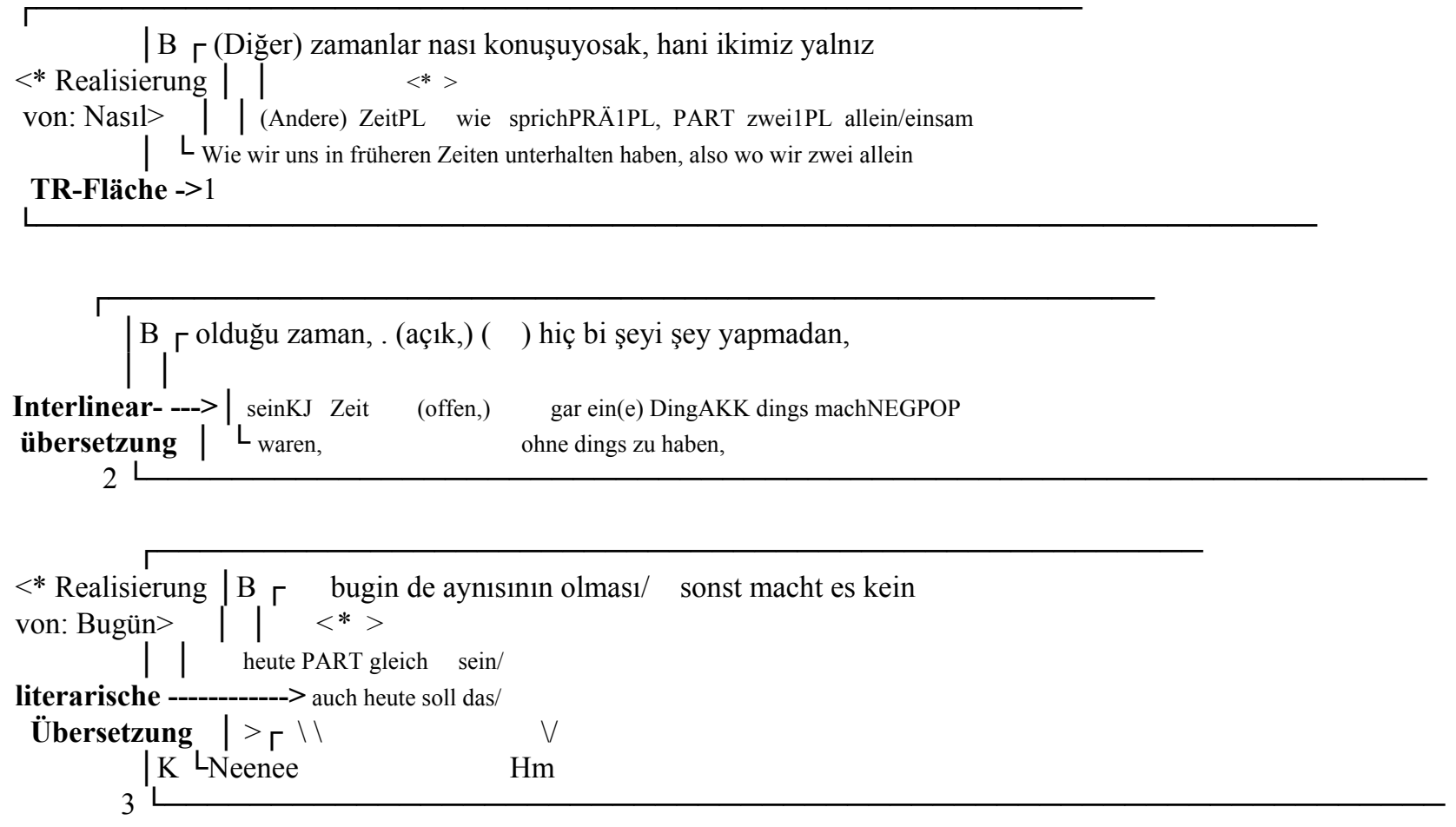

$\begin{gathered}\text { Sprechersigle }-> \\ \text { des Beraters }\end{gathered}$
4

\footnotetext{
Sprechersigle $->\mid \mathbf{K}[$ tu so als wärst Du mit mir alleine, obwohl das am des Klienten 5
}

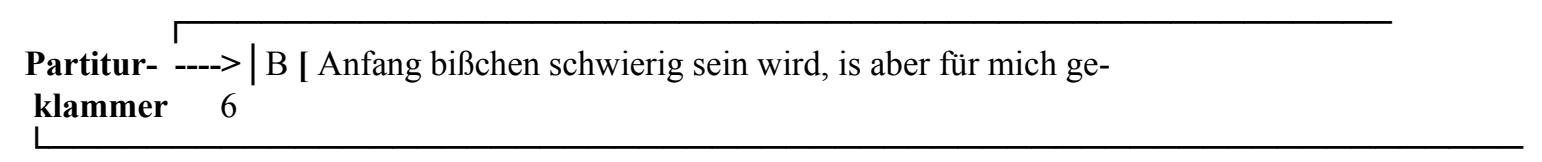




$$
\begin{aligned}
& \mathrm{B}_{\mathrm{L}} \Gamma \text { nauso schwierig. } \begin{array}{c}
\text { Und ich/ ((hustet)) }((2 \mathrm{~s})) \text { Gut. .. } \\
<\text { blättert----- }
\end{array}
\end{aligned}
$$

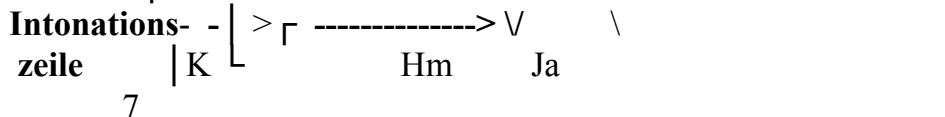

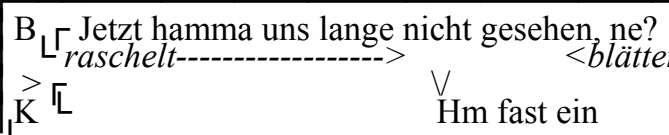

Wie oben in der Fläche 4 zu sehen ist, bekommt jeder Sprecher jeweils eine Sprechersigle (vgl. oben die Siglen B=Berater/Beraterin, $\mathrm{K}=$ Klient/Klientin). Redet lediglich eine Person im Diskurs länger, erhält die Partiturfläche nur eine Zeile (s.o. TR-Beispiel 1, Fl. 6). In den Partiturzeilen werden hauptsächlich verbale Äußerungen wiedergegeben. Kommt es aufgrund von nicht sprachlichen Handlungen zum Schweigen oder aktionalen Handlungen bei einem Sprecher, so wird die nicht sprachliche Handlung in Doppelklammern in der Partiturzeile für die verbale Kommunikation notiert, wie ((liest__ -$)$ ), ((sucht Stift --) etc. Dabei wird nicht Sprachliches vom zeitlichen Beginn bis zum zeitlichen Ende markiert (s.u. TR-

Beispiel 2, Fl. 23/24-Hervorhebung (S.D.), vgl. auch TR-Ausschnitt 3, Fl. 4).

Transkriptbeispiel 2: Nonverbale Phänomenbeschreibung (TR-18BT10)

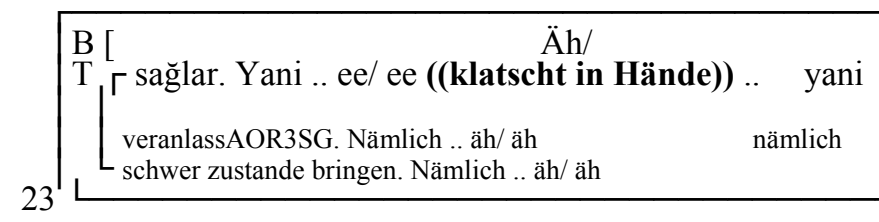

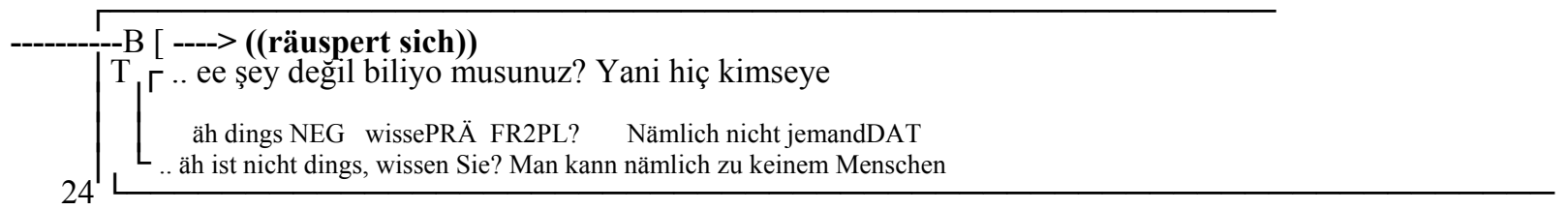


Ferner werden Kommentare für Paraverbalia wie z.B. 'leises, schnelles, ironis-ches' Sprechen in eckigen Klammern am Beginn und am Ende (einer Äußerung) gesetzt. Je nach technischer Möglichkeit werden Kommentare dieser Art entweder in der Partiturfläche bzw. Nonverbalzeile oder am linken Rand des Transkripts gemacht (s.u. TR 3, Fl. 5, 11 und 13-Hervorhebungen S.D.)

Transkriptbeispiel 3: Notation der Phänomenbeschreibung (TR-13BD3)

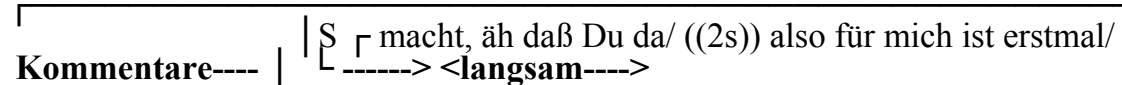




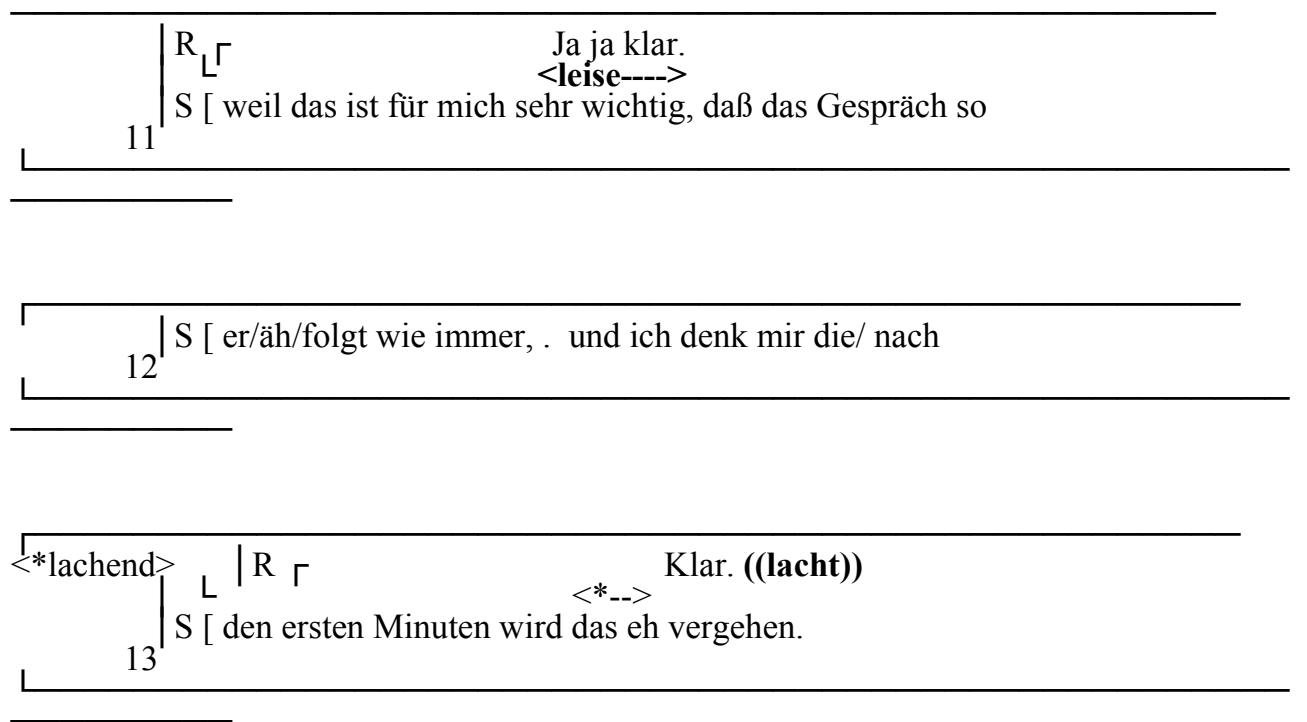

Die nonverbale Kommunikationszeile (vgl. TR-Beispiel 1, Fl. 2, 3) ermöglicht des Weiteren unter Beibehaltung der ursprünglichen Äußerungsreihenfolge die Interlinear- und sinngemäße Übersetzung. D.h. sowohl die wortwörtliche als auch die literarische Übersetzung in einer Fläche richtet sich nach der Originaläußerung. (s. dazu die hier angeführten Transkriptschnitte). HIAT-DOS wurde im Rahmen des erwähnten Forschungsprojektes 'Migrantensozialberatung' unter Leitung von Ehlich so weiterentwickelt, dass die Integration von Fonts für das Türkische und Serbokroatische und die Konvertierung bzw. technische Übertragung von DOS in die Word-Textverarbeitung möglich wurde.

Nach diesen Erläuterungen zum HIAT in ihren wichtigsten Grundzügen stellt sich die Frage nach der Lesbarkeit eines mit HIAT erstellten Transkripts. Man orientiert sich beim Lesen, wie unten die Pfeile am Transkriptausschnitt (TR-Beispiel 4) verdeutlichen, erstens an Links-rechtsLesung und das zweite Mal an der Oben-Unten-Oben-Lesung, d.h. der Leser liest von rechts nach links und von oben nach unten.

Transkriptbeispiel 4: Lesbarkeit des Transkripts (TR-3BT) 

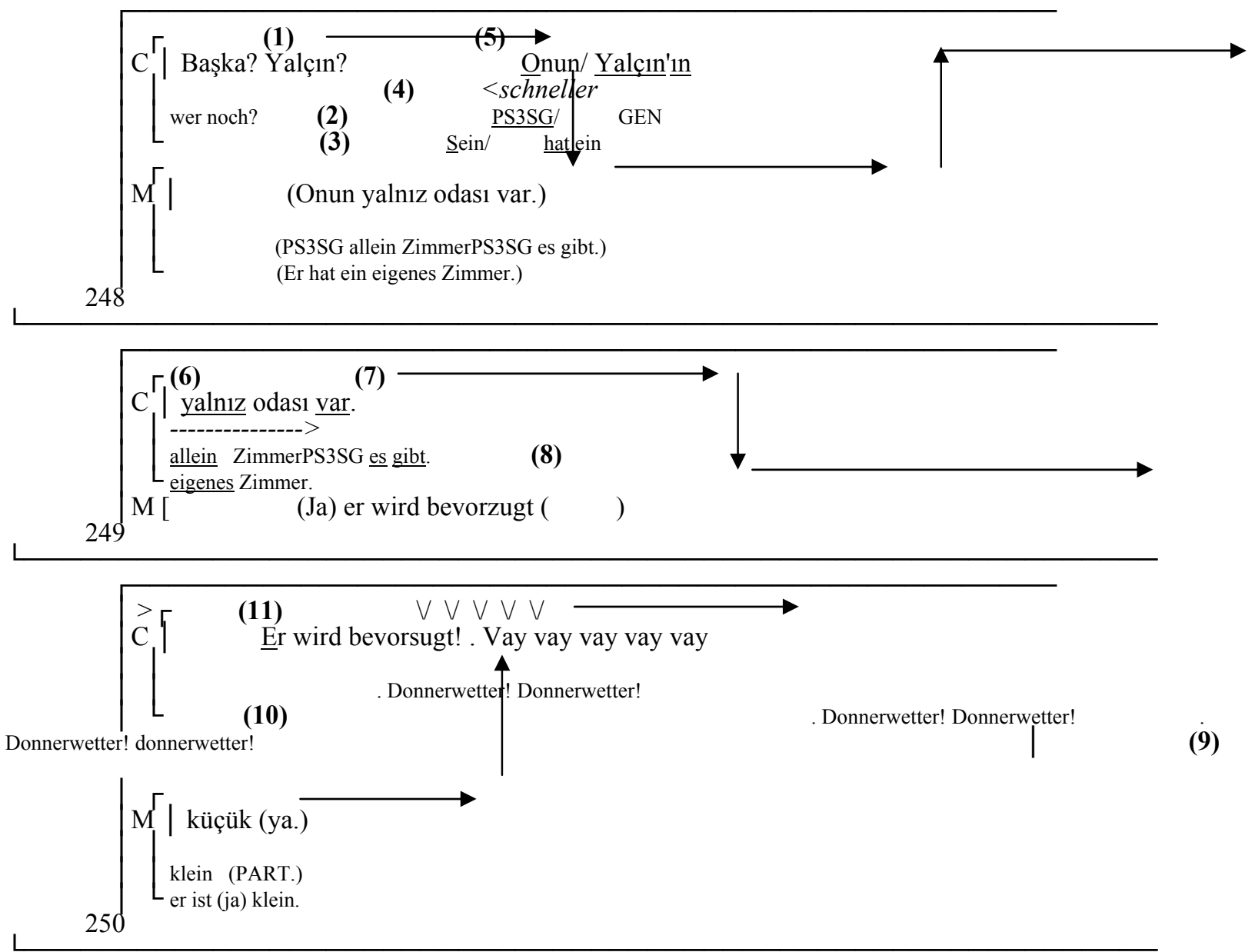

Über die hier beschriebenen Eigenschaften hinaus beinhalten HIATTranskripte Daten zum Aufnahmeort, zur Aufnahmezeit, zu Aufnahmeumständen und zum Transkribenten, die im Transkriptsigel festgehalten werden (s.u. Beispiel 5). Die Angabe zum Zeitverhältnis gibt die Relation des Mündlichen zu ihrer schriftlichen Umsetzung wieder: 1:120 bedeutet, dass für 1 Minute gesprochene Sprache 120

Minuten beim Transkribieren in Anspruch genommen wurden. HIAT ist ferner in kurzer Zeit erlernbar und durch Tastenfunktionen in DOS leicht handhabbar. Eigennamen von Beratern und Klienten sowie Ortsnamen sind in den Transkripten und auf dem Titelblatt (s.u. Beispiel 5) anonymisiert.

Beispiel 5: Ausgedrucktes Titelblatt eines Transkripts 


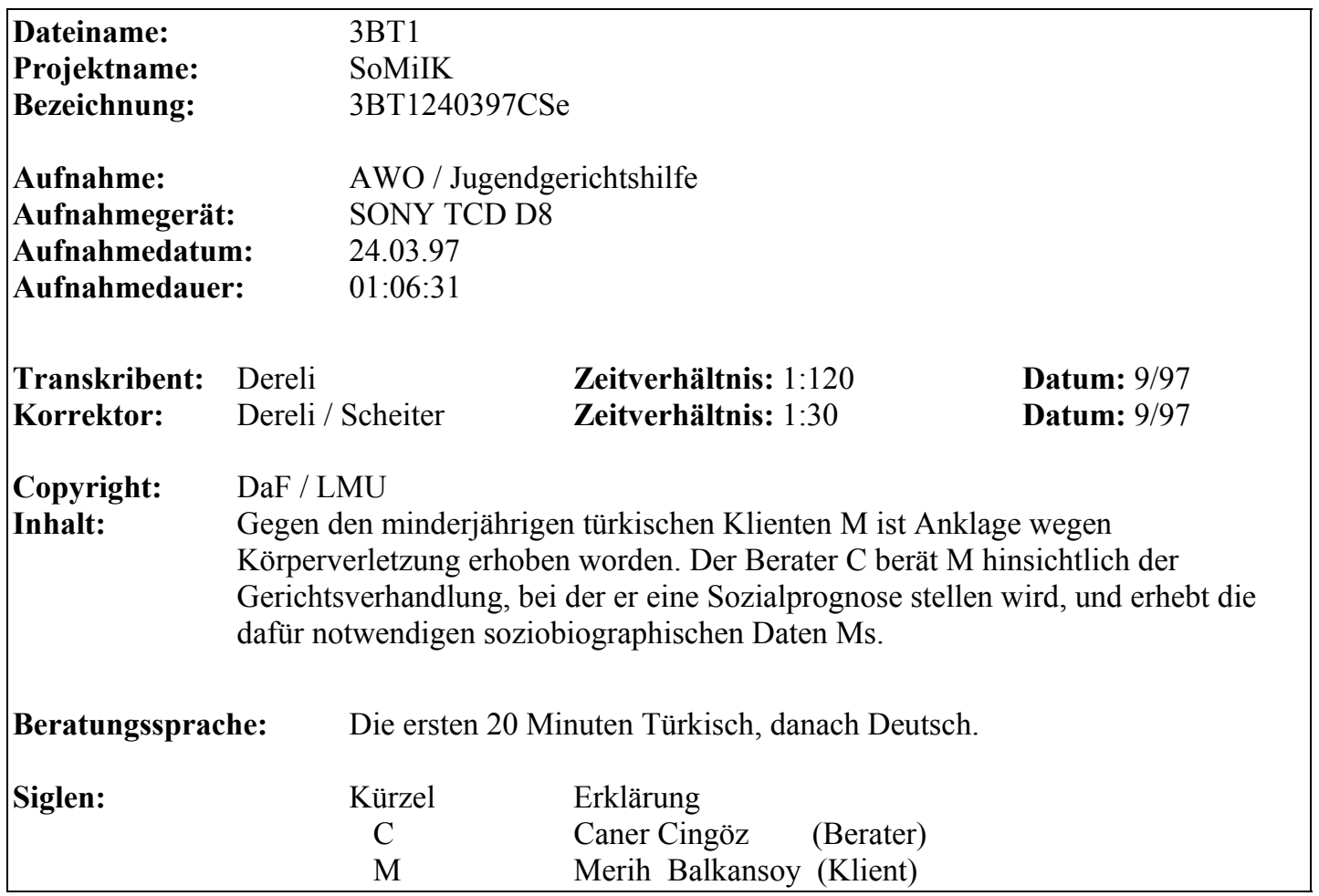

\section{Nutzen dieses Verfahrens in der Praxis}

Mit HIAT kann jede Tonband- und Videoaufzeichnung in Schriftzeichen umgesetzt und je nach Schwerpunkt des Untersuchungsgegenstandes hinsichtlich der verbalen Äußerungen, Paraverbalia und nonverbalen Handlungen exakt erfasst werden. Dieses Transkriptionsverfahren eignet sich für die Analyse von homileischen ${ }^{8}$ und institutionellen Diskursen. Die Verwendung der halbinterpretativen Arbeitstranskription beschränkt sich nicht nur auf die wissenschaftliche Erfassung eines 'Themas' hinsichtlich des analytischen Schwerpunktes, sondern sie kann zugleich praxisbezogen, z.B. in Kommunikationstrainings, aber auch in der Vermittlung des Deutschen als Fremdsprache, ${ }^{9}$ angewendet werden. Insofern sind Transkripte

Unter Rückbezug auf den griechischen Terminus homileo 'sich unterredend versammeln' (Ehlich/Rehbein 1980: 343) bezeichnen Ehlich und Rehbein mit homileisch Diskurse, die sich vom sprachlichen Handeln in Institutionen unterscheiden und zur Unterhaltung dienen. Dazu zählen z.B. Pausengespräche in der Schule sowie Unterhaltungen in Wartezimmern, Betrieben und 'der Schwatz beim Einkauf, am Brunnen, an der Pforte, bei der Arbeit usw. [...]' (ebd.).

9 Da es im Fremdsprachenunterricht sowohl um Vermittlung von Sprachstrukturen als auch um Vermittlung adäquater situationsbezogener Handlungskompetenzen geht, kann der Einsatz von Transkriptausschnitten die 
mit HIAT im Fremdsprachenunterricht sowohl im schulischen als auch im universitären Bereich einsetzbar und dienen somit einem 'reflektierten' Handeln aller Agierenden in Institutionen. Ihr Einsatz im Fremdsprachenunterricht besteht, wie Liedke-Göbel 2005 ausführt, in der Dokumentation des gesprochenen Deutsch (sowie andere Sprachen wie das Türkische), die man als Lehr- und Lernmaterial verwenden kann. Des Weiteren erfasst ein Transkript einen realen Ausschnitt aus der Wirklichkeit, sei es ein Beratungsdiskurs, ein Gespräch auf der Ausländerbehörde oder im Wohnungsamt usw., womit Lerner in ähnlichen Situationen im Zielland in ihrem alläglichen Handeln konfrontiert werden. Außerdem tragen verschriftlichte Daten zu Sprachvergleichen und zur Sprachstandsmessung mit Fehlerbehebung bei.

\section{Resümee}

In diesem Artikel wurden unterschiedliche Transkriptionssysteme in ihren Grundzügen dargestellt. Dabei wurde der Schwerpunkt auf das HIATTranskriptionssystem gelegt, das sich für die Analysen von gesprochener Sprache im Alltag und in Institutionen etabliert hat. Gekennzeichnet ist das HIAT-Transkriptionsverfahren, wie dargelegt, einerseits von der exakten Wiedergabe des Gehörten sowie von der Wiedergabe von parasprachlichen Mitteln und nonverbalen Phänomenen wie lachen, das z.B. im Diskurs funktional eine Reparaturhandlung darstellen kann und andererseits durch seine leichte Lesbarkeit auffällt.

$$
\begin{array}{ll}
\text { Verwendete Abkürzungen } \\
\begin{array}{ll}
\text { Fl. } & =\text { Fläche } \\
\text { GAT } & =\text { Gesprächsanalytische Transkription } \\
\text { HIAT } & =\text { Halbinterpretative Arbeitstranskription } \\
\text { s.u. } & =\text { siehe unten } \\
\text { s.o. } & =\text { siehe oben } \\
\text { TR } & =\text { Transkript }
\end{array}
\end{array}
$$

lernerseitigen 'Blicke' darauf schärfen, wie man mit Sprache und in Institutionen handelt. Zugleich kann sie 'Kommunikationsregeln' (z.B. in polizeilichen Verhören, ärztlichen Konsultationen, Beratungen etc.) für bestimmte Berufsgruppen wie Polizisten, Ärzte, Lehrer, Staatsanwälte, Psychologen etc. liefern und somit zur Erkenntnisgewinnung in der Praxis dienen. 


\section{Literatur}

Dereli, S./Kanz, R./Scheiter, S. 1998 Arbeitspapier Zum Transkribieren und Übersetzen von gesprochener Sprache - Erläuterungen zum Korpus „Migrantenberatungen“. München: mimeo (Institut für DaF/TnG).

Austin, J. L. $2002^{2}$. Zur Theorie der Sprechakte (How to do Things with Words). Ditzingen: Reclam.

Becker-Mrotzek, M. 1994. Diskursforschung in der alten BRD. In: Ehlich, K. (Hg.): Diskursanalyse in Europa. Frankfurt a. Main u.a.: Lang, 87 - 107 (Forum angewandte Linguistik, Bd.24).

Ehlich, K./Rehbein, J. 1976. Halbinterpretative Arbeitstranskriptionen (HIAT). In: Linguistische Berichte 45, 21 - 41.

Ehlich, K./Rehbein, J. 1979. Erweiterte halbinterpretative Arbeitstranskriptioneb 2. In: Linguistische Berichte 59, 51 - 75.

Ehlich, K./Rehbein, J. 1981. Die Wiedergabe intonatorischer, nonverbaler und aktionaler Phänomene im Verfahren HIAT. In: Lange-Seidl, A. (Hg.): Zeichenkonstitution, Bd. 2, Berlin: de Gruyter, 174 - 186.

Ehlich, K./Schwitalla, B. 1976. Transkriptionssysteme - Eine exemplarische Übersicht. In: Studium Linguistik 2, 78 - 103.

Ehlich, K./Rehbein, J. 1986. Muster und Institution. Untersuchungen zur schulischen Kommunikation, Tübingen: Narr.

Ehlich,K./Rehbein, J. 1980². Sprache in Institutionen. In: Althaus, H.P. (Hg.): Lexikon der germanistischen Linguistik, Tübingen: Niemeyer, 338 - 345.

Grießhaber, W. 1987. Authentisches und zitierendes Handeln. Einstellungsgespräche und Rollenspiele im Sachunterricht, Tübingen: Narr.

Hoffmann, L. (Hg.) 1989. Rechtsdiskurse. Tübingen: Narr.

Iványi, Z. (1996). Die ethnomethodologische Konversationsanalyse. Eine darstellende Beschreibung. In: Sprachtheorie und germanistische Linguistik 3, 61-89.

Liedke-Göbel, M. 2005. Transkription. Einführung mit praktischen Übungen, Institut für Deutsch als Fremdsprache/Transnationale Germanistik der LudwigMaximilians-Universität (Skript: Sommersemester 2005).

Löning, P./Sager, S. (Hg.) 1986. Kommunikationsanalysen ärztlicher Gespräche. Hamburg: Buske.

Redder, A. 1984. Modalverben im Unterrichtsdiskurs. Tübingen: Niemeyer.

Rehbein, J. 1986. Institutioneller Ablauf und interkulturelle Missverständnisse in der Allgemeinpraxis. Diskursanalytische Aspekte der Arzt-PatientenKommunikation. In: Curare 9, 297 - 328. 
Schlobinski, P. 1996. Empirische Sprachwissenschaft. Opladen: Westdeutscher Verl.

Searle, R. J. 1983. Sprechakttheorie. Ein sprachphilosophischer Essay, Frankfurt am Main: Suhrkamp.

Wunderlich, D. (Hg.) 1972. Linguistische Pragmatik. Frankfurt / Main: Athenäum.

Wunderlich, D./ Maas, U. $1972^{2}$. Pragmatik und sprachliches Handeln, Frankfurt / Main: Athenäum.

\section{Online-Quellen}

HIAT und HIAT-DOS: http://www.daf.uni-muenchen.de/HIAT/HIAT.HTM.

Knöbel, R./Steiger, K.: Transkription: Transkriptionssysteme. http://www.idsmannheim.de/prag/GAIS/ transsysteme.php

Schneider, W. 2001. Der Transkriptionseditor HIAT-DOS. In: Gesprächsforschung Online-Zeitschrift zur verbalen Interaktion, 2, $29 \quad-\quad 33$ (http://www.gespraechsforschung-ozs.de) 